\title{
Revista Brasileira de Enfermagem REBEn Qualidade de vida e severidade da doença em idosos renais crônicos
}

\author{
Quality of life and measure of disease in elderly people with end-stage renal disease \\ Calidad de vida y severidad de la enfermedad en ancianos con Insuficiencia Renal \\ Crónica Terminal
}

Fabiana Ferreira de Souza

Enfermeira. Mestre em Enfermagem pelo Programa de Pós-Graduação em Enfermagem da Faculdade de Ciências Médicas da UNICAMP - Campinas - São Paulo.

Fernanda Aparecida Cintra

Enfermeira. Professora Doutora do Departamento de Enfermagem da Faculdade de Ciências Médicas da UNICAMP - Campinas - São Paulo. fernanda@fcm.unicamp.br

Maria Cecília B. Jaime Gallani

Enfermeira. Professora Doutora do Departamento de Enfermagem da Faculdade de Ciências Médicas da UNICAMP - Campinas - São Paulo.

Trabalho extraído da dissertação de mestrado: Souza FF. Avaliação da qualidade de vida do idoso em hemodiálise: comparação de dois instrumentos genéricos [dissertação]. Campinas (SP): Departamento de Enfermagem da Faculdade de Ciências Médicas; UNICAMP; 2003.

\section{RESUMO}

O objetivo da pesquisa foi verificar, em uma população de idosos com Insuficiência Renal Crônica Terminal (IRCT) em hemodiálise ambulatorial, a relação entre uma medida genérica de qualidade de vida (WHOQOLbreve) e a medida de severidade da IRCT (ESRD-SI - Índice de Severidade da IRCT). Trata-se de um estudo correlacional de corte transversal, realizado em duas clínicas de terapia renal substitutiva, com 100 idosos portadores de IRCT, em tratamento hemodialítico há pelo menos seis meses. A análise mostrou que os domínios do WHOQOL-breve correlacionaram-se negativamente com a severidade da ICRT, exceto os domínios psicológico e meio ambiente. Além disso, todos os domínios do WHOQOL-breve mostraram poder de discriminar os sujeitos em relação à severidade da doença.

Descritores: Insuficiência Renal Crônica; Diálise renal; Psicometria.

\section{ABSTRACT}

The purpose of this research was to identify, in an elderly population with end-stage renal disease (ESRD) undergoing ambulatory hemodialysis, the correlation between a generic index of quality of life (WHOQOL-BREF) and the severity index of the ESRD-SI. The work consisted of a correlational cross-section study conducted at two clinics providing replacement of renal therapy and involved 100 elderly patients suffering from ESRD, who had already been in hemodialytic treatment for at least six months. The WHOQOL-BREF showed a negative correlation with the severity of the ESRD-SI and the technique employed allowed for a distinction to be made between patients with greater and lesser severity of ERSD-SI.

Descriptors: Kidney Failure, chronic; Renal dialysis; Psychometrics.

\section{RESUMEN}

El objetivo de la investigación fue verificar en una población de ancianos con Insuficiencia Renal Crónica Terminal (IRCT) en hemodiálisis en seguimiento ambulatorio la relación entre una medida genérica de cualidad de vida (WHOQOL -breve) y la medida de la severidad de la IRCT (ESRD-SI - Índice de la Severidad de la IRCT). ES un estudio que establece correlaciones y de cohorte transversal que fue desarrollado en dos clínicas de terapia renal substitutiva, con 100 ancianos portadores de IRCT, en programa de hemodiálisis en periodo superior a seis meses. EI WHOQOL -breve estableció una correlación negativa con la severidad de la IRCT (ESRD-SI) y permitió distinguir los ancianos con relación a la severidad de la IRCT.

Descriptores: Insuficiencia Renal Crónica; Diálisis renal; Psicometría.

Souza FF, Cintra FA, Gallani MCBJ. Qualidade de vida e severidade da doença em idosos renais crônicos. Rev Bras Enferm 2005 set-out; 58(5):540-4.

\section{INTRODUÇÃO}

Nos últimos 20 anos observa-se um crescimento expressivo no número de idosos com insuficiência renal, o que ocorre concomitante ao número de co-morbidades ${ }^{(1)}$.

No passado, a terapia renal substitutiva consistia numa terapêutica excludente da população idosa, visto que o seu acesso era restrito por fatores econômicos e sociais, associados à sua menor expectativa de vida (2). Esse panorama vem se modificando no decorrer dos anos, conforme os dados da Sociedade Brasileira de Nefrologia, que em 2001 registrou 48.806 pacientes em tratamento dialítico no Brasil. Desse total, aproximadamente um terço apresentava idade superior a 60 anos.

A proposta da terapia renal substitutiva nos idosos fundamenta-se na melhora da sua Qualidade de Vida (QV), resgatando-lhes o bem-estar físico e a capacidade cognitiva, além de mantê-los inseridos no contexto social ${ }^{(2)}$. Essa proposta, entretanto, esbarra nas alterações da vida diária que o tratamento hemodialítico desencadeia, pela falta de suporte familiar e da equipe de saúde para a 
manutenção do tratamento.

Os idosos em tratamento dialítico geralmente apresentam maior comprometimento funcional, o que remete à necessidade de assistência em suas atividades de vida diária ${ }^{(3)}$. Associado ao comprometimento físico, os pacientes renais crônicos, e em particular os idosos, costumam sofrer alterações no desempenho de seus papéis sociais e dos aspectos psicológicos decorrentes da situação do adoecer ${ }^{(4,5)}$.

Em estudo comparativo entre o comprometimento funcional, a depressão e a satisfação com a vida entre idosos portadores de Insuficiência Renal Crônica Terminal (IRCT) em hemodiálise e um grupo controle de idosos moradores na comunidade os autores ${ }^{(6)}$ mostraram $^{2}$ que os idosos em hemodiálise apresentaram comprometimento funcional mais significativo, escores de depressão mais elevados, assim como menor satisfação com a vida. Esses achados fornecem subsídios para ratificar a compreensão de que a Insuficiência Renal Crônica (IRC), e a sua terapêutica, geram forte impacto negativo na vida dos pacientes, acometendo inúmeras dimensões da qualidade de vida ${ }^{(5,7)}$.

Assim, a análise da QV do idoso com IRCT em tratamento hemodialítico revela-se importante para orientar medidas de intervenção que contemplem prioritariamente os aspectos mais comprometidos da QV neste grupo de sujeitos e também os não comprometidos, visando a sua manutenção.

Dessa forma, é desejável que os instrumentos de medida de QV sejam multidimensionais e capazes de abranger grande parte dos aspectos presentes no constructo $\mathrm{QV}$, ou aqueles mais relevantes para a população em estudo.

A maioria dos estudos que abordam o paciente idoso com IRCT tem empregado instrumentos genéricos para a medida da QV relacionada à saúde.

Dentre os instrumentos genéricos disponíveis, o WHOQOL-breve, desenvolvido pelo grupo de estudos de QV da Organização Mundial de Saúde (OMS), apresenta como ponto forte a inclusão de um domínio para a avaliação da QV relacionada ao meio-ambiente, o qual tende a ser valorizado pelo idoso(8)

No Brasil, o WHOQOL-breve $\mathrm{e}^{(9,10)}$ foi utilizado em estudo que relacionou a QV com a capacidade funcional de um grupo de idosos, e mostrou-se adequado para a população idosa ${ }^{(11)}$. Recentemente, em outro estudo realizado em Taiwan os autores ${ }^{(12)}$ verificaram a adequação deste instrumento para a população de idosos moradores em uma área residencial, e concluíram que o WHOQOL-breve pode ser considerado um instrumento de medida de QV adequado aos idosos, desde que sejam realizadas pequenas modificações.

$\mathrm{Na}$ avaliação da QV relacionada à saúde não apenas a medida do constructo deve ser objetivada, mas também dos fatores sobre os quais se faz uma assumpção hipotética de relação estreita com a QV, ou de influência sobre o seu curso. Na literatura é freqüente 0 relato do emprego de instrumentos que buscam medir constructos afins da medida da QV, como, por exemplo, escalas de medida de depressão, escalas de grau de independência funcional, severidade percebida ou impacto da doença, bem como de avaliações sistemáticas da condição clínica dos sujeitos.

Na avaliação clínica do paciente, em particular do portador de uma doença crônica, como a IRCT, acredita-se na importância dos profissionais de saúde conhecerem a gravidade da afecção, ou melhor, avaliarem se as complicações físicas trazidas por essa afecção, e que correspondem à sua severidade, estão associadas à QV.

Neste contexto, foi construído o End - Stage Renal Disease - Severity Index (ESRD-SI), que contempla as doenças mais comuns em pacientes renais crônicos como Diabetes mellitus, doença cardiovascular, entre outras. Esse instrumento é preenchido pelo profissional médico que assiste 0 paciente, e corresponde a uma medida objetiva para caracterizar a gravidade global da IRCT ${ }^{(13)}$.

Considerando-se, portanto, que a pesquisa da influência (ou associação) de fatores objetivos, como a severidade da doença sobre a QV, pode fornecer subsídios importantes para o direcionamento de intervenções que levem à melhora da QV, o presente estudo teve como objetivo geral: verificar a relação entre uma medida genérica de QV e a medida de severidade da doença, em um grupo de idosos com IRCT em programa de hemodiálise ambulatorial. Os objetivos específicos foram: 1. Testar a correlação entre a medida de QV e de severidade da IRCT; e, 2. Comparar a medida da QV, mensurada pelo WHOQOL-breve, entre os sujeitos distribuídos em duas categorias de severidade da IRCT.

\section{CASUÍSTICA E MÉTODO}

\subsection{Sujeitos e Local}

No estudo participaram idosos com idade igual ou superior a 60 anos, com diagnóstico de IRCT, em programa de hemodiálise ambulatorial, atendidos em duas clínicas de terapia renal substitutiva (hemodiálise) do Estado de São Paulo, nas cidades de Campinas e São Carlos, no período de janeiro a março de 2003.

Constituíram critérios de inclusão: estar em programa de hemodiálise há pelo menos seis meses; apresentar capacidade de compreensão e de comunicação verbal; e concordar em participar da pesquisa, com a assinatura do Termo de Consentimento Livre e Esclarecido. Os critérios de exclusão foram: apresentar alterações cognitivas ou distúrbios psiquiátricos; ser portador de neoplasias; ou ter antecedência pessoal de transplante renal.

Considerando os valores de coeficiente de correlação $r \geq 0,40$, e de $\alpha=0,05$ e $\beta=0,05$, estimou-se um número mínimo de 75 sujeitos para compor a amostra. Como esse número foi muito próximo do total de sujeitos da população alvo, optou-se por incluir todos os idosos, das duas clínicas eleitas para essa investigação, que atendiam aos critérios de inclusão e nenhum dos de exclusão, totalizando 100 sujeitos.

Os 100 idosos estudados caracterizaram-se pelo equilíbrio entre os sexos (51,0\% do sexo masculino); idade entre 60 e 86 anos (média = $68,3 \pm 6,4)$; tempo médio de escolaridade de 3,2 anos de estudo $( \pm 3,4$; mediana $=3,0)$; e predomínio da cor branca $(81,0 \%)$. A maioria dos pacientes $(67,0 \%)$ possuía um parceiro. Quanto à renda mensal familiar informada, a média foi 3,3 salários mínimos $(\mathrm{SM})( \pm 5,2$; mediana =2,0), oscilando entre 0,5 e 42 SM.

O tempo médio de tratamento hemodialítico dos pacientes foi 26,7 meses $( \pm 21,7$; mediana $=20,5)$, com variação entre seis e 117 meses. As condições clínicas associadas que se mostraram mais prevalentes no grupo estudado foram: Diabetes mellitus tipo 2 (42,0\%) e hipertensão arterial sistêmica $(79,0 \%)$. Estas condições também corresponderam ao fator etiológico da IRCT para $38,0 \%$ e $22,0 \%$ dos sujeitos, respectivamente. Outras condições clínicas freqüentes, como 0 déficit visual e a insuficiência cardíaca, destacaram-se em $25,0 \%$ e 15,0\% dos pacientes, respectivamente. Uma proporção significativa de idosos $(45,0 \%)$ apresentava duas ou três condições clínicas associadas.

\subsection{Instrumentos}

\subsubsection{WHOQOL-breve}

O WHOQOL-breve, desenvolvido por um Grupo da Organização Mundial da Saúde em $1998^{(9)}$ e validado no Brasil em $2000^{(10)}$, consta de 26 questões, das quais duas são gerais sobre QV e as 24 restantes abordam quatro domínios: físico, psicológico, relações sociais e meio ambiente.

No presente estudo os valores de coeficiente alfa de Cronbach $(\alpha)$ obtidos na aplicação do WHOQOL-breve revelaram os seguintes valores: instrumento global $=0,87$; domínio físico $(D F)=0,79$; domínio psicológico $(D P)=0,63$; domínio relações sociais $(D R S)=0,42 ;$ e, domínio meio ambiente $(D M A)=0,69$. Este desempenho mostrou-se 
semelhante ao que tem sido descrito na literatura nacional e internacional(10-12)

2.2.2 ESRD - SI (End Stage Renal Disease - Severity Index) Índice de Severidade da Insuficiência Renal Crônica Terminal

O ESRD-SI, desenvolvido nos Estados Unidos da América em 1991 ${ }^{(13)}$ e validado no Brasil em $2002^{(14)}$, é composto de 11 categorias que incluem as doenças, suas complicações, e as condições mais comuns do paciente renal crônico, como: insuficiência cardíaca, acidente vascular cerebral, doença vascular periférica, neuropatia periférica, neuropatia autonômica e distúrbios gastrointestinais, acesso e eventos da diálise, Diabetes mellitus, entre outras.

A classificação desse instrumento corresponde a valores numéricos, calculados proporcionalmente para cada categoria, e obtém em sua somatória um índice de gravidade global, que pode variar de zero a 94 . Nesse índice, "zero" representa a ausência de doenças associadas (além da IRC), e 94 corresponde à severidade grave da IRC, tendo em vista a associação de todas as condições clínicas comumente manifestas no renal crônico em suas formas mais graves, previstas no instrumento.

\subsection{Procedimento}

Inicialmente foi realizada uma entrevista individual com os idosos, previamente à terapia renal substitutiva (hemodiálise) ou, na sua impossibilidade, nas duas primeiras horas do tratamento, uma vez que o paciente com freqüência apresenta alterações hemodinâmicas após este intervalo de tempo. Nessa entrevista foi aplicado o WHOQOL-breve sob a forma de entrevista, considerando-se a possibilidade dos sujeitos apresentarem queda da acuidade visual e baixo nível instrucional.

Posteriormente foi solicitado ao médico nefrologista, que atendia os idosos, o preenchimento do ESRD-SI (Índice de Severidade da Insuficiência Renal Crônica Terminal) de cada idoso.

O estudo foi formalmente autorizado pelos diretores das respectivas clínicas de nefrologia, bem como aprovado pelo Comitê de Ética em Pesquisa da Faculdade de Ciências Médicas da Universidade Estadual de Campinas - UNICAMP (Parecer CEP nº 417/2002)

\subsection{Análise Estatística}

A análise estatística foi realizada com 0 apoio do Serviço de Estatística da Comissão de Pesquisa da Faculdade de Ciências Médicas da UNICAMP.

Os dados coletados foram inicialmente transportados para uma planilha de dados do programa Excel for Windows 98 e, então, para o programa SAS System for Windows (Statistical Analysis System) versão 8.02, para as seguintes análises: 1. descritiva (tabelas de freqüência, medidas de posição e dispersão, bem como os casos válidos e omitidos); 2. de correlação (coeficiente de Correlação de Spearman, para verificar a correlação entre os instrumentos WHOQOL-breve e ESRD-SI); e, 3. de comparação (Teste de Mann-Whitney, para verificar o poder de discriminação dos itens do WHOQOL-breve em relação a severidade da IRCT- ESRD-SI). O nível de significância adotado para os testes estatísticos foi de $5 \%$.

\section{RESULTADOS}

A avaliação da gravidade da insuficiência renal crônica terminal, por meio do ESRD - SI, nas categorias originais, mostrou que $87,0 \%$ dos casos de IRCT foram categorizados como sendo de grau suave de severidade, $12,0 \%$ como grau suave e moderado, e somente $1,0 \%$ como grau moderado. A média do escore do ESRD-SI foi $14,0( \pm 9,9)$, com mediana $=14,0$. Estes dados revelam um grupo homogêneo de gravidade da doença renal, configurada como suave para a maioria dos casos, de acordo com a avaliação pelo ESRD-SI.

Como houve uma grande concentração dos pacientes na categoria suave (87\%), e uma dispersão entre as categorias suave/moderada e moderada, optou-se neste estudo pela aglutinação das duas últimas categorias, dando origem à seguinte classificação: "suave" para os idosos que obtiveram pontuação $\leq 24$ e "não-suave" para os idosos com pontuação $>24$. Na Tabela 1 é apresentada a distribuição dos sujeitos de acordo com as categorias do ESRD-SI.

Tabela 1. Distribuição dos 100 sujeitos de acordo com as categorias do ESRD-SI. Campinas, 2003.

\begin{tabular}{lclc}
\hline ESRD - SI & & ESRD - SI & \\
Categorias Originais & $\%$ & Categorias Modificadas & $\%$ \\
\hline Suave & 87,0 & Suave & 87,0 \\
Suave / Moderada & 12,0 & Não-Suave ** & 13,0 \\
Moderada & 1,0 & & \\
Moderada / Grave & -- & & \\
Grave & -- & & 100,0 \\
\hline TOTAL & 100,0 & & \\
\hline
\end{tabular}

* pontuação $\leq 24$; ** pontuação $>24$

A Tabela 2 mostra que o ESRD-SI correlacionou-se negativamente com os domínios físico e relações sociais da QV, avaliados pelo WHOQOL-breve, sendo estas correlações de moderada magnitude. Embora tenha sido observada correlação negativa entre a severidade e os domínios psicológico e meio ambiente, estas correlações apresentaram somente uma tendência de significância estatística.

Tabela 2. Coeficiente de Correlação de Spearman dos escores dos domínios do WHOQOL-breve com o ESRD-SI. Campinas, 2003.

\begin{tabular}{lc}
\hline Domínios do WHOQOL-breve & ESRD-SI \\
\hline DF & $p=-0,37$ \\
& $p=0,0001$ \\
DP & $r=-0,20$ \\
& $p=0,0509$ \\
DRS & $r=-0,22$ \\
& $p=0,0254$ \\
DMA & $r=-0,19$ \\
\hline
\end{tabular}

DF= Domínio Físico; DP= Domínio Psicológico; DRS= Domínio Relações Sociais; DMA= Domínio Meio Ambiente. $r=$ Coeficiente de Correlação de Spearman; $p=p$-valor.

Na Tabela 3 é descrita a comparação entre os itens do WHOQOLbreve e as duas categorias do ESRD-SI (suave e não-suave) criadas neste estudo. Nota-se que o WHOQOL-breve conseguiu discriminar os sujeitos com severidade suave e não-suave da IRCT em todos os domínios.

Tabela 3. Comparação entre os valores dos escores obtidos nos quatro domínios do WHOQOL-breve entre os sujeitos com ESRDSI Suave $(n=87)$ e Não-suave ( $n=13)$ (Teste de Mann-Whitney). Campinas, 2003.

\begin{tabular}{|c|c|c|c|c|}
\hline $\begin{array}{l}\text { Oominios do } \\
\text { WHOQOL-breve }\end{array}$ & & $\begin{array}{l}\text { ESRO-ST } \\
\text { Suave }\end{array}$ & $\begin{array}{c}\text { ESRDD-ST } \\
\text { Nao-Suave }\end{array}$ & p-valor \\
\hline DF & $\begin{array}{l}\text { Media } \\
\text { (Edp) } \\
\text { Mediana }\end{array}$ & $\begin{array}{c}53,4( \pm 18,0) \\
57,1\end{array}$ & $\begin{array}{c}36,5( \pm 10,0) \\
35,7\end{array}$ & $p=0,0009$ \\
\hline$D P$ & $\begin{array}{l}\text { Media } \\
\text { (Edp) } \\
\text { Medians }\end{array}$ & $\begin{array}{c}62,3(\neq 13,8] \\
62,5\end{array}$ & $\begin{array}{c}51,0( \pm 15,2) \\
58,3\end{array}$ & $p=0,0297$ \\
\hline DRS & $\begin{array}{l}\text { Media } \\
\text { (Idp) } \\
\text { Medians }\end{array}$ & $\begin{array}{c}66,0( \pm 11,0\} \\
65,7\end{array}$ & $\begin{array}{c}54,5( \pm 13,9) \\
58,3\end{array}$ & $p=0,0057$ \\
\hline DMA & $\begin{array}{l}\text { Media } \\
\text { (Idp) } \\
\text { Medians }\end{array}$ & $57,5 \leqslant \neq 12,4\}$ & 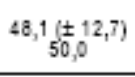 & $p<0,0208$ \\
\hline
\end{tabular}

$D F=$ Domínio Físico; $D P=$ Domínio Psicológico; $D R S=$ Domínio Relações Sociais; $D M A=$ Domínio Meio Ambiente. $p=p$-valor.

Esses dados revelam que embora não tenha havido uma correlação linear entre todos os domínios do instrumento WHOQOL-breve e a severidade da doença, ao se comparar os sujeitos com severidade suave e severidade não-suave observou-se que em todos os domínios houve diferença na QV, apontando o grupo de severidade não-suave como 0 mais comprometido na medida da QV. 


\section{DISCUSSÃO}

\subsection{A severidade da IRCT na vida do idoso}

Conforme apontado anteriormente, o ESRD-SI foi desenvolvido com o propósito de avaliar, por meio de uma variável de controle, a severidade da doença renal crônica terminal com a mínima influência das variáveis psicossociais ${ }^{(13)}$. O fato de não incluir em sua estrutura variáveis psicossociais torna o ESRD-SI um instrumento útil para testar hipóteses psicossociais ${ }^{(15)}$.

A análise dos dados da presente investigação indica que a maioria dos sujeitos obteve um índice de severidade médio de 14,0 ( $\pm 9,9), 0$ qual é considerado suave. Do total de sujeitos estudados, $87,0 \%$ tiveram a sua doença categorizada como de severidade suave, ou seja, houve uma homogeneidade dos idosos em relação ao ESRD-SI.

Em estudo realizado com pacientes submetidos a diversas formas de tratamento dialítico, obteve-se uma pontuação média do ESRD-SI da ordem de 10,2. Especificamente na faixa etária entre 63 e 80 anos, esse índice médio subiu para 12,4 $4^{(13)}$.

No presente estudo, a média do escore do índice de severidade da IRCT foi equivalente ao índice médio $(15,5)$ registrado em pacientes submetidos a diálise peritoneal intermitente, e mostrou um valor superior ao registrado nos pacientes em tratamento hemodialítico $(8,1)^{(13)}$.

Embora o ESRD-SI tenha mostrado originalmente propriedades satisfatórias, chama a atenção a grande concentração de sujeitos na categoria suave, tanto no presente estudo como naqueles apontados na literatura ${ }^{(13,15)}$.

Essa homogeneidade na severidade da IRCT pode, de certa forma, apontar para uma falta de sensibilidade do instrumento para distinguir os sujeitos portadores dessa afecção que se enquadram na categoria suave. A não discriminação de peculiaridades dos sujeitos pertencentes a esta categoria, por sua vez, pode limitar análises futuras, que busquem estabelecer correlação entre severidade e outras variáveis.

Acredita-se que um refinamento do ESRD-SI, que possibilitasse a melhor discriminação dos sujeitos na categoria suave, poderia resultar numa maior sensibilidade nas correlações entre a severidade da doença e as variáveis psicossociais.

4.2 A correlação e a comparação entre a QV (WHOQOL-breve) e a severidade da IRCT

Com respeito à severidade, todos os domínios do WHOQOL-breve discriminaram os sujeitos com severidade suave e não-suave da IRCT, embora apenas dois de seus domínios tenham apresentado correlação negativa significativa com a pontuação da severidade: o domínio físico, com moderada magnitude, e o domínio relações sociais, com fraca magnitude. Os domínios psicológico e meio ambiente exibiram somente uma tendência à fraca correlação negativa.

É preciso destacar que, em relação à severidade da IRCT, apesar da maioria dos sujeitos $(87,0 \%)$ ter sua doença categorizada como de severidade suave, todos os domínios do WHOQOL-breve foram capazes de discriminar os idosos com severidade suave ou não-suave.

$\mathrm{Na}$ literatura nacional e internacional identificam-se poucas investigações que correlacionam a severidade da IRCT com a QV dos pacientes renais crônicos.

Na aplicação de três medidas de QV em pacientes em diálise: a técnica "time trade-off ", a QV relacionada à saúde e o SF-36, e com o emprego do ESRD-SI, os autores encontraram correlação negativa entre o ESRDSI e os componentes físico e mental do SF-36, correspondendo a forte $(r=-0,60 ; p$-valor $=0,0001)$ e moderada magnitude $(r=-0,39 ; p$-valor= $0,0033)$, respectivamente ${ }^{(16)}$.

Os dados do presente estudo apontam para a existência de correlação com magnitude mais acentuada entre a severidade da doença e o aspecto físico da QV, em relação ao aspecto mental, ratificando os achados da literatura ${ }^{(15,17)}$.

\section{CONCLUSÃO}

Finalizando, a análise desta pesquisa em um grupo de pacientes renais crônicos idosos em hemodiálise ambulatorial permite concluir: 1) os domínios do WHOQOL-breve correlacionaram-se negativamente com a severidade da ESRD-SI, exceto os domínios psicológico e meio ambiente, que apresentaram apenas uma tendência significativa estatisticamente; e, 2) todos os domínios do WHOQOLbreve tiveram poder de discriminar os sujeitos em relação à severidade da doença.

Estes achados e a escassez de literatura sobre o tema remetem a futuros estudos em outros grupos e culturas a fim de possibilitar as respectivas comparações, e outras que forneçam elementos importantes para orientar medidas de intervenção nos domínios da QV que se mostram comprometidos nesse grupo populacional. Remetem, ainda, a estudos longitudinais que avaliem o efeito das intervenções clínicas sobre a medida da QV desses sujeitos.

\section{REFERÊNCIAS}

1. Pires AJ. Insuficiência renal. In: Freitas EV, Py L, Neri AL, Cançado FAX, Rocha SM, organizadores. Tratado de Geriatria e Gerontologia. Rio de Janeiro (RJ): Guanabara Koogan; 2002. p. 457-66.

2. Carvalho FJW, Silva ABF, Costa RC. Avaliação da diálise no paciente idoso. Arq Geriatria Gerontol 1999 jan; 3(1): 5-10.

3. Mckevitt PM, Jones JF, Lane DA, Marion RR. The elderly on dialysis: some considerations in compliance. Am J Kidney Dis 1990 out; 4:346-50.

4. Fonseca PP. Um questionário para a avaliação do impacto de doenças crônicas: tradução validada e estudo de confiabilidade do IEQ (IIIness Effects Questionnaire) [dissertação]. São Paulo (SP): Departamento de Enfermagem, Universidade Federal de São Paulo; 2001.

5. Silva DMGV, Vieira RM, Koschnik Z, Azevedo M, Souza SS. Qualidade de vida de pessoas com insuficiência renal crônica em tratamento hemodialítico. Rev Bras Enferm 2002 set-out; 55(5): 562-67.

6. Kurtner NG, Brogan D, Hall D, Haber M, Daniels DS. Functional impairment, depression, end life satisfaction among older hemodialysis patients and age-matched controls: a prospective study. Arc Phys Med Rehab 2000 abr; 81: 453-9.

7. Valderrábano F, Jofre R, López-Gómez, JM. Quality of life in endstage renal disease patients. Am J Kidney Dis 2001 set; 38(3):443-64.
8. Lawton MP, Windley PG, Byerts TO. Aging and the environment: theoretical approaches. New York (USA): Springer, 1982.

9. The WHOQOL Group. Development of the World Health Organization WHOQOL-bref. Quality of life assessment. Psych Med 1998; 28:551-8.

10. Fleck MPA, Leal OF, Louzada S, Xavier M, Chachamovich E, Vieira $G$, Santos $L$ et al. Aplicação da versão em português do instrumento abreviado de avaliação da qualidade de vida "WHOQOL- bref'. Rev Saúde Pública 2000 abr; 34(2): 178-83.

11. Geib TM. Associação entre a capacidade funcional e qualidade de vida de idosos do Município de São Paulo, residentes na comunidade que moram sozinhos [dissertação]. São Paulo (SP): Departamento de Enfermagem, Universidade Federal de São Paulo; 2001.

12. Hwang HF, Liang WM, Chiu YN, Lin MR. Suitability of the WHOQOLbref for community-dwelling older people in Taiwan. Age and Ageing, 2003 jun; 32: 593-600.

13. Craven J, Littefield C, Rodin G, Murray M. The end-stage renal disease severity index (ESRD-SI). Psych Med 1991; 21: 237-43.

14. Fonseca PP, Oliveira AFF, Del Porto JA. Uma escala para a avaliação clínica padronizada de pacientes com IRC Terminal: tradução validada e estudo da confiabilidade do ESRD-SI (End Stage Renal 
Souza FF, Cintra FA, Gallani MABJ.

Disease - Severity Index). In: Anais do $21^{\circ}$ Congresso Brasileiro de Nefrologia e $11^{\circ}$ Congresso Brasileiro de Enfermagem em Nefrologia; 2002 set 90; Brasília (DF), Brasil. Brasília (DF): ABEn; 2002.

15. Griffin $\mathrm{K} \mathrm{W}$, Friend $\mathrm{R}$, Wadhwa NK. Measuring disease severity in patients with end-stage renal disease: validity of the Craven et al. ESRD Severity Index. Psych Med 1995; 25: 189-93.
16. Maor Y, King M, Olmer L, Mozes B. A comparison of three measures: the time trade-off technique, global health-related quality of life and the SF-36 in dialysis patients. J Clin Epidemiol 2001; 54: 565-70.

17. Rodin T, Voshart K. Depression in the medically ill: an overview. Am J Psych 1986; 696-705. 